\title{
Performance evaluation of the next generation solid-state digital photon counting PET/CT system
}

\author{
Jun Zhang ${ }^{1}$, Piotr Maniawski ${ }^{2}$ and Michael V. Knopp ${ }^{*^{*}}$ D
}

\begin{abstract}
Background: The first solid-state silicon photomultiplier (SiPM) digital photon counting (DPC) clinical PET/CT system was introduced by Philips in recent years. The system differs from other SiPM-based PET/CT systems and uses lutetiumyttrium oxyorthosilicate (LYSO) scintillators directly coupled with their own individual SiPM DPC detectors eliminating the need for Anger-logic positioning decoding. We evaluated the system performance, characteristics, and stability of the next generation DPC clinical PET/CT based on NEMA NU2-2012 tests, NEMA NU2-2018 test (timing resolution) and human studies.
\end{abstract}

Results: An energy resolution of $11.2 \%$ was measured. NEMA NU2-2012 tests revealed a spatial resolution (mm in FWHM) from $(3.96,4.01,4.01)$ at $1 \mathrm{~cm}$ to $(5.81,5.83,4.95)$ at $20 \mathrm{~cm}$ for (axial, radial, tangential). A $5.7 \mathrm{cps} / \mathrm{kBq}$ system sensitivity was measured. Peak noise equivalent count rate (NECR) and peak true count rate could not be determined as each exhibited increasing values up to the maximum activity measured ( $1100 \mathrm{MBq})$. The maximum NECR was $171 \mathrm{kcps} @ 50.5 \mathrm{kBq} / \mathrm{mL}$, with corresponding scatter fraction of $30.8 \%$ and maximum trues of $681 \mathrm{kcps}$. NEMA hot sphere contrast ranged from $62 \%(10 \mathrm{~mm})$ to $88 \%(22 \mathrm{~mm})$, cold sphere contrast of $86 \%(28 \mathrm{~mm})$ and $89 \%$ (37 mm). A timing resolution of 322 ps ( ${ }^{22} \mathrm{Na}$ point source based) and $332 \mathrm{ps}$ (NEMA NU2-2018) was obtained. It revealed $<1 \%$ change in TOF timing and $\pm 0.4 \%$ change in energy resolution during 31 -month stability monitoring. CQIE assessment found $<3 \%$ axial variance in SUV. 100-60\% recovery coefficients of activity concentration at various sphere sizes and contrast levels were measured.

Conclusions: This scanner represents the first solid-state DPC PET/CT, a technologic leap beyond photomultipliers tubes and anger logic. It presents considerable improvements in system performance and characteristics with excellent time-of-flight capability compared to conventional photomultiplier tube (PMT) PET/CT systems. The DPC system leads to promising clinical opportunities with excellent image quality, lesion detectability, and diagnostic confidence.

Keywords: Digital photon counting, Solid state, Silicon photomultiplier, Positron emission tomography, System performance

\section{Background}

PET/CT has evolved to be an essential imaging modality in oncology and neuroscience. Up to now, PET detectors have been mainly based on photomultiplier tubes (PMT) which have advantages of high gain signal amplification, multichannel capabilities and good timing performance,

\footnotetext{
* Correspondence: knopp.16@osu.edu

${ }^{1}$ Wright Center of Innovation in Biomedical Imaging, Department of Radiology, The Ohio State University Wexner Medical Center, 395 W. 12th Avenue, Room 430, Columbus, OH 43210, USA

Full list of author information is available at the end of the article
}

however have also disadvantages of limited photon-to-electron quantum conversion efficiency and relative bulkiness [1, 2]. Recent efforts pushed beyond these limitations to further improve PMT PET/CT system performance [3-5].

Modern solid-state detectors were developed in the 90s using silicon metal oxide semiconductor (MOS) structures with avalanche breakdown mode operation capable of detecting single light photons [6]. An initial concept of Geiger-mode avalanche photodiodes (APDs) or silicon photomultipliers (SiPMs) was proposed in the 
late 90s combining with many features of PMTs and traditional APDs [7]. Analog SiPM detectors started to be available for medical applications around 2004 [8] and were introduced to more imaging applications with relative compact design and better MR compatibility however initially without time of flight (TOF) capabilities [9-16]. Analog SiPM detectors using light-sharing designs use single photon avalanche diode (SPAD) arrays to detect single scintillation photons, while the pulses generated by multiple SPADs are combined into one analog output signal requiring off-chip processing and anger logic decoding [17].

Philips Healthcare introduced the first solid-state, SiPM digital photon counting (DPC) PET/CT system at RSNA 2013. Before that, its prototype system demonstrated better image quality and diagnostic accuracy compared with Gemini TF PET [18]. The DPC detector differs from analog SiPM detectors: each SPAD is part of an array running as a digital counter, and the readout of the digital signal provides a direct count of the number of scintillation photons detected without analog signal processing $[17,19]$. The system uses lutetiumyttrium oxyorthosilicate (LYSO) scintillators directly coupled with individual SiPM DPC detectors eliminating the need for Anger-logic positioning decoding [20, 21].

This medical physics focused study evaluates the DPC system performance characteristics in sensitivity, noise equivalent counting rate (NECR), spatial resolution, image quality, energy resolution, and TOF timing resolution using National Electrical Manufacturers Association (NEMA) NU2-2012 and the recent NU2-2018 as well as other methodologies.

\section{Methods}

\section{System specifications}

The Vereos PET/CT (dPET) (Philips, Cleveland OH) is a system based on SiPM DPC technology coupled with a 64-slice helical CT of the Ingenuity class, and its first system was operational at Wright Center of Innovation in Biomedical Imaging (WCIBMI) at The Ohio State University (OSU) since 07/2014. This system fulfilled all system specifications of the commercially released system (10/2017) and was used to perform clinical trials for regulatory submissions. The CT uses a $40 \mathrm{~mm}$ axial field of view (FOV) with 3D dose modulation for low dose CT capabilities integrated with an iterative reconstruction technique (iDose ${ }^{4}$ ) and the Metal Artifact Reduction for Orthopedic Implants algorithm. The $764 \mathrm{~mm}$ PET detector ring spans $164 \mathrm{~mm}$ in the axial FOV and is composed of 18 flat detector modules with 4 by 5 array SiPM detector tiles on each module. Every tile consists of a $4 \times 4$ matrix of sensor silicon dies, and each die is a $2 \times 2$ matrix of digital photon counter detectors (silicon pixels). Every pixel couples directly to a $3.86 \times$
$3.86 \times 19 \mathrm{~mm}^{3}$ single LYSO crystal for a total of 23,040 crystal-DPC pairs. 3200 SPAD (microcells) are integrated on each pixel resulting in $73,728,000$ microcells that enable detection of single scintillation photons with each photon being converted directly into a pure binary output signal (Fig. 1). The number of microcells recording a photon is read out as the total number of photons detected by a pixel.

PET data are acquired in 3D mode and stored in a list-mode file containing position, timing, and energy information of each DPC-collected event. PET reconstruction is performed using a list-mode-based TOF OSEM algorithm with blobs as basic functions [22, 23]. It uses a Monte Carlo-based scatter simulation for scatter correction [24] and Casey averaging for smoothed randoms estimation [25]. The system applies $2.0 \mathrm{~ns}, 4.0 \mathrm{~ns}$, and $4.6 \mathrm{~ns}$ coincidence windows depending on the transaxial FOVs $(256 \mathrm{~mm}$ for brain, $576 \mathrm{~mm}$ and $676 \mathrm{~mm}$ for body). PET reconstruction uses isotropic voxels for standard definition (SD) $\left(4 \times 4 \times 4 \mathrm{~mm}^{3}\right)$, high definition (HD) $\left(2 \times 2 \times 2 \mathrm{~mm}^{3}\right)$ and ultra-high definition (UHD) $\left(1 \times 1 \times 1 \mathrm{~mm}^{3}\right)$. Post-reconstruction image processing includes an optional Richardson-Lucy, maximum-likelihood resolution recovery implementation and Gaussian filtering [26-28].

\section{Digital photon counting (DPC)}

The DPC technology allows every microcell to be individually activated or inactivated, SPADs and data processing are integrated onto a single silicon chip at each pixel allowing fast and accurate ultra-low light single photon detection, and each microcell directly detects and counts the breakdown of an individual SPAD on its silicon chip. A light photon is detected by one of the integrated 3200 microcells yielding a pure binary signal measured by the on-chip photon counter and timer. Background noise, or say dark count rate (DCR), is measured and managed effectively for each individual counter. The biggest contribution to the overall sensor DCR is caused by only a small percentage of the cells (such as $\sim 10 \%$ cells responsible for $70-80 \%$ DCR). Disabling high dark count rate cells on a small active sensor area leads to significantly reduced overall sensor DCR; therefore, the overall DCR can be greatly reduced by switching off the noisiest cells. Each sensor operates independently from the other sensors. Only those die sensors that detect sufficient photons reaching the configured thresholds will start the acquisition sequence (Fig. 2). In the beginning, a die sensor is in the "ready state," where all its microcells are fully charged, and the system waits for the start of the photon events until a trigger occurs. When the number of photons detected in a silicon pixel becomes higher than the configured threshold, it prompts a timestamp to be saved and 


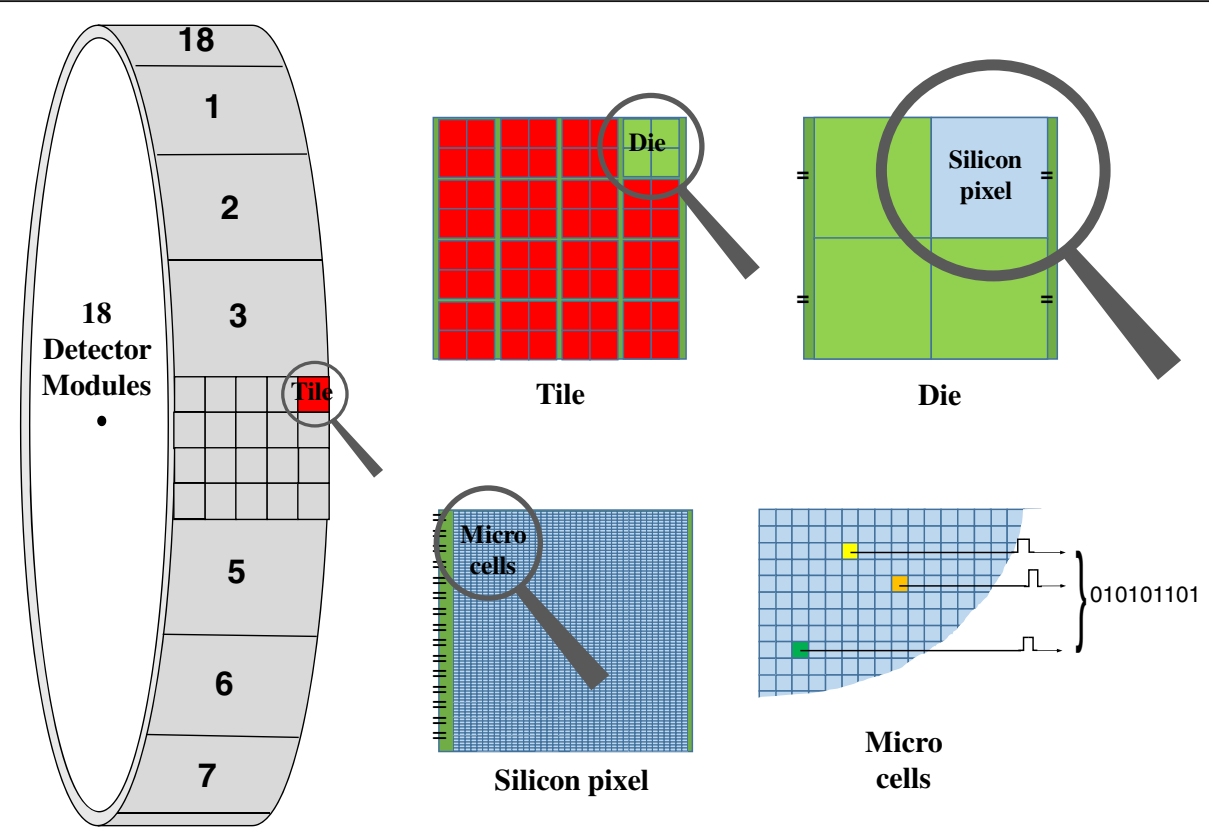

Fig. 1 Solid-state digital photon counting PET detector design. When a scintillation photon hits the microcell sensor, the integrated photon counter increases and the integrated timer measures the arrival time of the individual photon on that die. The chip measures all photons during the desired duration of the detection process. The values of the integrated photon counter and timer are read out via a digital interface

begins a validation process to detect a user-configured number of further photons within a certain time. If this validation threshold is exceeded, there is a subsequent integration period before a readout process sends data (four photon count values, one per silicon pixel on the die, and one timestamp per event) to a readout buffer. After readout, the microcells are recharged so that the die is ready for further data acquisition. If the validation threshold was never reached, all microcells are immediately recharged and go back to the "ready state." At the end, the photon counter is read out by summing overall detected photons with a timestamp, and the whole process is entirely digital without need of signal amplification. The localization determination of the scintillation light created by gamma-ray interaction using the DPC compared to traditional PMT-based approach is shown in Fig. 3.

\section{System performance measurements}

System performance measurements of the dPET utilized NEMA NU 2-2012 procedures [29] for spatial resolution, sensitivity, scatter fraction, count loss, NECR, and image quality.

Spatial resolution of a PET scanner is an intrinsic feature of the device, which reflects the ability of the system to distinguish between two points after image reconstruction, and is usually measured in transaxial (radially and tangentially) and axial directions. Spatial resolution of the system was measured using a point source of $4 \mathrm{MBq}{ }^{18} \mathrm{~F}$ in a capillary tube (tube inside diameter $\leq 1 \mathrm{~mm}$, tube length $=100 \mathrm{~mm}$, axial extend of the activity: approximately $1 \mathrm{~mm}$ ). Measurements were performed at the center of axial FOV and three-eighths of the axial FOV from the center of the FOV with $(x, y)$ locations of $(0,1),(0,10)$, and $(0,20) \mathrm{cm}$. For the axial resolution measurements, we rotated the capillary with $90^{\circ}$ which was perpendicular to the long axis of the system so that the axial extent of the source was within the diameter of the capillary ( $1 \mathrm{~mm}$ or less). List-mode data were reconstructed using 3D Fourier re-projection with an unapodized filter [30]. Pixel size of the reconstructed images used for spatial resolution calculation was $1 \times 1 \times 1 \mathrm{~mm}^{3}$. Axial, radial, and tangential resolutions in full width at half-maximum (FWHM) and full width at tenth-maximum (FWTM) for each radius (1, 10 , and $20 \mathrm{~cm}$ ), averaged over both axial positions were calculated and reported.

Sensitivity of PET represents the ability to detect true coincidence events in $\mathrm{cps} / \mathrm{kBq}$, which typically depends on solid angle, system photon detection efficiency, dead time and other factors, and is measured based on established techniques [31, 32]. NEMA sensitivity was measured at the center and $10 \mathrm{~cm}$ off in transverse FOV using five aluminum sleeves (Data Spectrum Corp., Hillsborough, NC; $70 \mathrm{~cm}$ in length, inner diameters 3.9$16.6 \mathrm{~mm}$ ) and a length of $70 \mathrm{~cm}$ plastic tubing with $6 \mathrm{MBq}{ }^{18} \mathrm{~F}$. The initial activity was calibrated in a dose calibrator which in general has less than $1 \%$ accuracy 


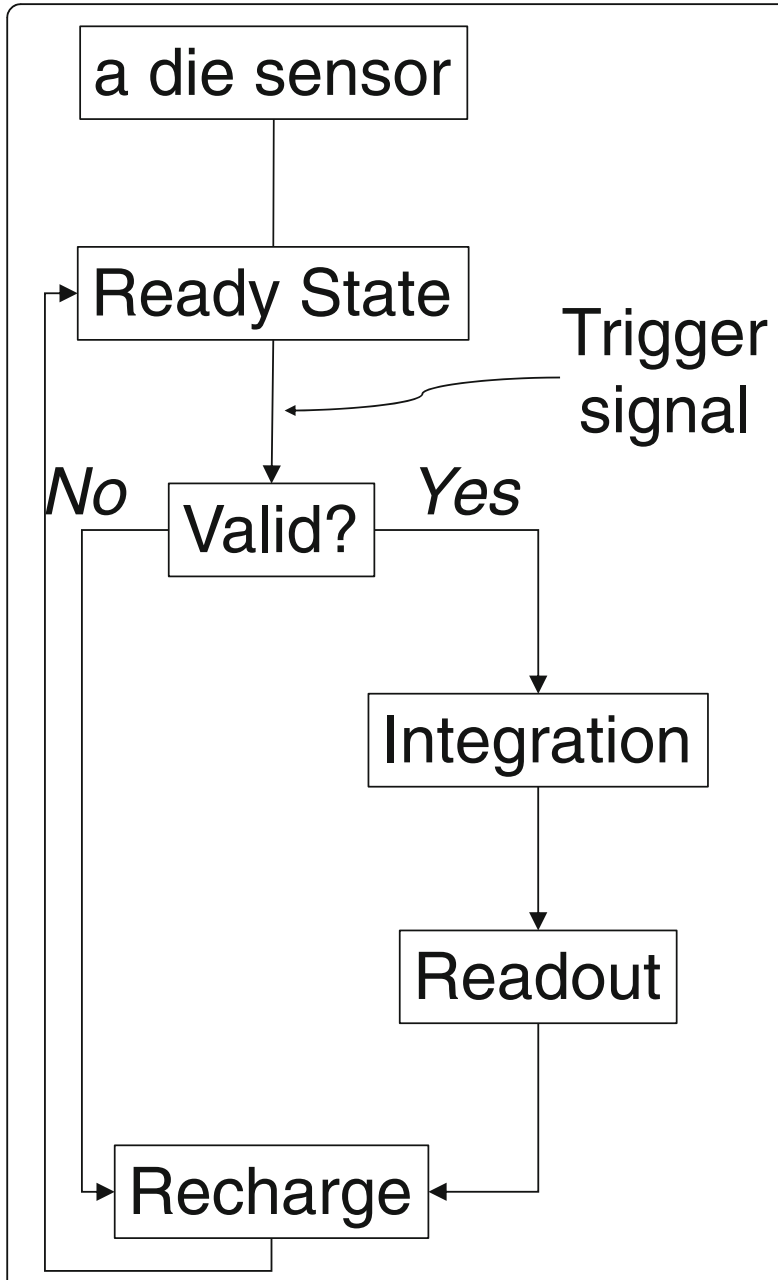

Fig. 2 Single event-based data acquisition sequence on each die sensor of the investigated DPC PET system

error. Successive measurements were performed $10 \mathrm{~min}$ post ${ }^{18} \mathrm{~F}$ injection by adding aluminum sleeves of known attenuation. Decay-corrected count rate was summed for all slices to give the total count rate for each sleeve and then extrapolated to an attenuation free measurement.

Measurements of scatter fraction, count losses, and NECR were performed using the NEMA PET scatter phantom. A plastic tubing (3.2 $\mathrm{mm}$ inner diameter) filled with $\sim 1100 \mathrm{MBq}{ }^{18} \mathrm{~F}$ over a length of $70 \mathrm{~cm}$ was measured over 16-h until the activity decayed to a low level with true events losses of $<1 \%$. Data were binned into sinograms and used to calculate the scatter fraction and count rates.

For measurements of image quality and scatter corrections, a NEMA IEC Body phantom with ${ }^{18} \mathrm{~F}(75.9 \mathrm{MBq})$ was abutted with a $70 \mathrm{~cm}$ scatter cylinder phantom containing ${ }^{18} \mathrm{~F}$ line source $(140 \mathrm{MBq})$ to approximate the clinical situation of having activity extending beyond the scanner. The imaging phantom contains two cold spheres (28 and $37 \mathrm{~mm}$ ) and four hot spheres (10$22 \mathrm{~mm}$ ), positioned circular with a radius of $57.2 \mathrm{~mm}$ at the center of the phantom similar to NEMA NU 2-2012 standard. The sphere-to-background ratio (SBR) was 4 . Acquisition was repeated three times with $180 \mathrm{~s} / \mathrm{bed}$. Contrast for each hot sphere $\left(Q_{H}\right)$ and each cold sphere $\left(Q_{C}\right)$ was calculated as

$$
Q_{H}=100 \% \times \frac{C_{M, S} / C_{M, B}-1}{C_{T, S} / C_{T, B}-1} \text { and } Q_{C}=100 \% \times\left(1-\frac{C_{M, S}}{C_{M, B}}\right) .
$$

where $C_{M, S}$ and $C_{M, B}$ are the averaged activity concentration in a circular ROI with a diameter equal to the inner diameter of the sphere $(S)$ being measured $(M)$ or in the background $(B)$ and $C_{T, S}, C_{T, B}$ are the true $(T)$ activity concentration.

The lung error was calculated by averaging the relative lung errors of each slice in axial direction according to the NEMA NU2-2012 definition.

Energy and timing resolution are not part of the standard NEMA NU2-2012 testing. The coincidence timing resolution as a function of activities was measured using the NEMA NEC phantom data according to NEMA NU2-2018 standard and its associated methodologies [33-35]. For the stability monitoring, energy and timing resolution were additionally measured using built-in PET daily quality check (QC) procedures. Energy window was set at $88-120 \%$ of $511 \mathrm{keV}(450-613 \mathrm{keV})$, and the coincidence-timing window was set at $4 \mathrm{~ns}$ with a delayed coincidence window technique utilized to estimate the random coincidences. A $3.7 \mathrm{MBq}{ }^{22} \mathrm{Na}$ point source centered in PET FOV was used with daily acquisition of PET coincidence ( 200 Mcounts). Energy and timing resolution were then calculated separately from event histograms with $4 \mathrm{keV}$ bins for energy and 19.6 ps bins for timing. In addition, sensitivity as a function of activities was separately measured using the NEMA NEC phantom data [34, 35].

\section{System stability}

System stability of sensitivity (in relative count rate), energy resolution, and timing resolution were monitored over a 31-month period between October 2014 and May 2017. These parameters were extracted from the built-in PET daily QC and corresponding system log files.

\section{PET image quality assessment}

PET image quality was additionally evaluated for uniformity, partial volume effect (PVE), maximum recovery coefficients $\left(\mathrm{RC}_{\max }\right)$, and clinical lesion detectability.

PET uniformity assessment was performed according to the NCI Center for Quantitative Imaging Excellence (CQIE) program guidelines [36]. A $9293 \mathrm{~mL}$ water cylinder phantom with $53 \mathrm{MBq}$ FDG was scanned and 


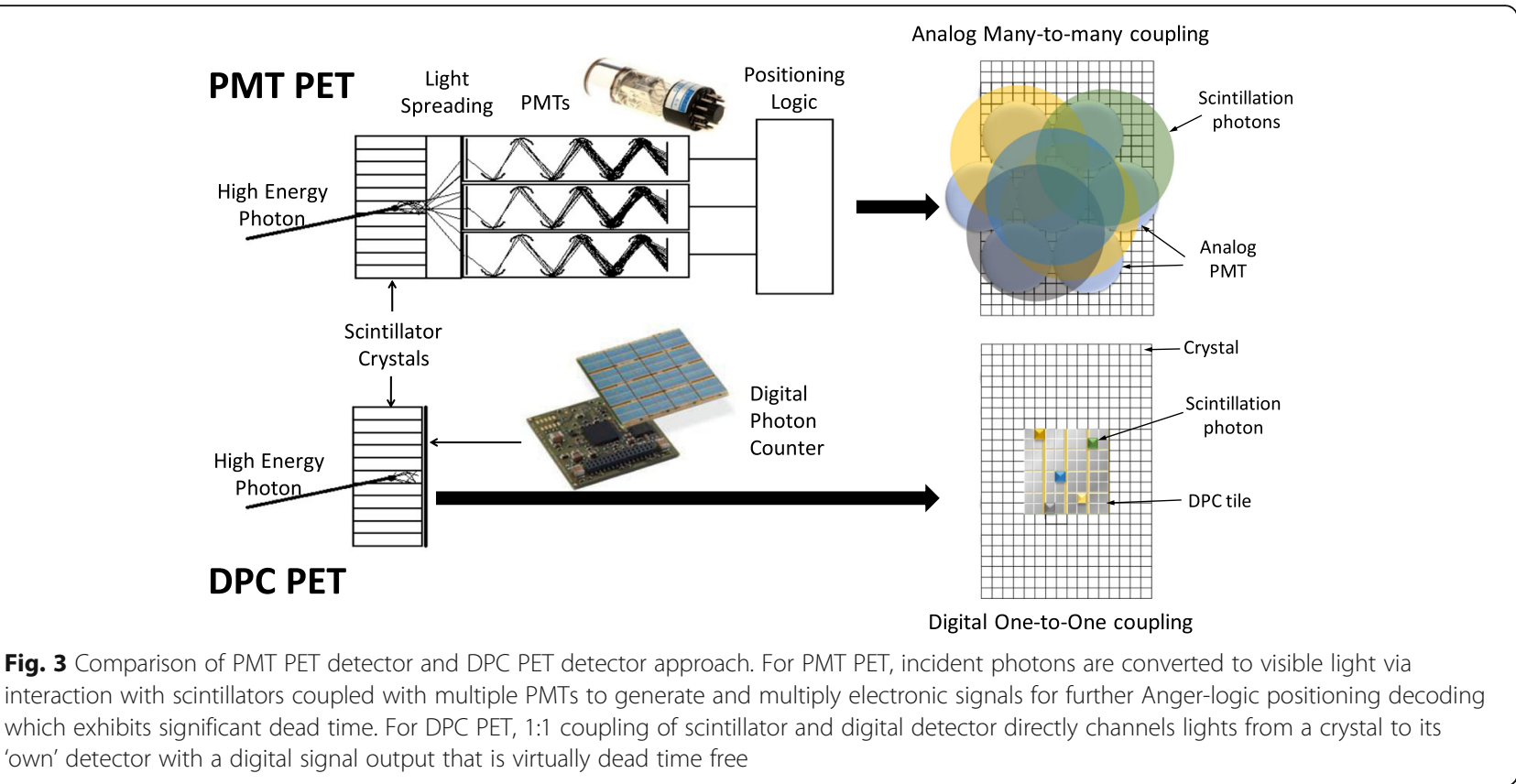

reconstructed using 3D OSEM TOF. A 2D circular region-of-interest (ROI, $\sim 200 \mathrm{~cm}^{2}$ ) was placed across all slices. Images were analyzed according to CQIE criteria (SUVmean of $0.9-1.1$ with $\leq 10 \%$ variances in axial).

For PVE assessment, a Jaszczak Flangeless Esser PET phantom with rod inserts $(4.8-12.7 \mathrm{~mm})$ was imaged at $60 \mathrm{~min}$ post $48 \mathrm{MBq}{ }^{18} \mathrm{~F}$-FDG injection with $90 \mathrm{~s} / \mathrm{bed}$ for body and $10 \mathrm{~min}$ for brain according to ACR accreditation guidelines [37]. Images were reconstructed in SD, HD, and UHD with different voxel sizes.

The phantom procedures different from the NEMA NU 2-2012 standard are detailed below for assessment of RC: a NEMA IEC Body phantom $(185 \pm 3.7 \mathrm{MBq}$ ${ }^{18}$ F-FDG) filled with 6 hot spheres $(10-37 \mathrm{~mm})$ and varying SBRs of 2, 4, 8, 16, 32, 64, and 100 was imaged with $75 \mathrm{~s} /$ bed and reconstructed in SD $\left(4 \times 4 \times 4 \mathrm{~mm}^{3}\right)$ using 3D OSEM TOF (3i15s for dPET and 3i33s for cPET). Spherical ROIs with approximately $37 \mathrm{~mm}$ in diameter were placed on each of the spheres. $\mathrm{RC}_{\max }$ was analyzed and defined as

$$
\mathrm{RC}_{\max }=\frac{C_{M, S}}{C_{T, S}}
$$

where $C_{M, S}$ is the maximum of the measured $(M)$ activity concentration in each sphere, and $C_{T, S}$ is the true $(T)$ activity concentration for sphere ROIs.

Assessments of clinical image quality and lesion detectability were performed based on PET/CT scans of clinical patients. PET/CT scans were acquired with $90 \mathrm{~s} /$ bed on the $\mathrm{APET}$ before or after local standard of care
PET/CT on a conventional PMT-based Gemini TF 64 system (cPET, $90 \mathrm{~s} / \mathrm{bed}, 75 \mathrm{~min}$ uptake, $481 \mathrm{MBq}$ ${ }^{18}$ F-FDG). All data were reconstructed using 3D TOF OSEM with 3 iterations (subset of 15 for SD dPET, and 33 for SD cPET). dPET CT was reconstructed using iDose $^{4}$ in $4 \mathrm{~mm}$ and used for PET attenuation correction. $\mathrm{SUV}_{\text {peak }}$ values of representative lesions were calculated using MIMSoftware 6.7 (MIM Software Inc.). The study (NCT02283125) was approved by the institutional review board, and all subjects signed an informed consent.

\section{Results}

System performance measurements and system stability

NEMA spatial resolution, sensitivity, NECR, count rate, scatter fraction, image quality, and timing resolution as well as the system stability in timing resolution, energy resolution and sensitivity are presented.

Table 1 demonstrates the spatial resolution of the dPET system, ranging from (in FWHM) $3.96 \mathrm{~mm}$ (axial), $4.01 \mathrm{~mm}$ (radial), and 4.01 (tangential) at the center toward the periphery of $5.81 \mathrm{~mm}$ (axial), $5.83 \mathrm{~mm}$ (radial), and $4.95 \mathrm{~mm}$ (tangential) at $20 \mathrm{~cm}$ off center.

The average NEMA sensitivity of the dPET system was $5721 \mathrm{cps} / \mathrm{MBq}$ at the center and $5637 \mathrm{cps} / \mathrm{MBq}$ at a $10 \mathrm{~cm}$ radial offset from the FOV center. Figure 4a details the measured NEMA sensitivity profile along axial slices, and Fig. 4b shows the stability of system sensitivity over 31 months (better than $1.5 \%$ variability) by monitoring the relative count rate as a surrogate for sensitivity compared to baseline. 
Table 1 NEMA spatial resolution of the dPET system

\begin{tabular}{|c|c|c|c|c|c|c|c|c|c|c|c|c|}
\hline \multicolumn{13}{|l|}{ Spatial resolution } \\
\hline & \multicolumn{6}{|c|}{ Measurements $01^{a}$} & \multicolumn{6}{|c|}{ Measurements 02} \\
\hline & FWHM & & & FWTM & & & FWHM & & & FWTM & & \\
\hline Direction & $1 \mathrm{~cm}$ & $10 \mathrm{~cm}$ & $20 \mathrm{~cm}$ & $1 \mathrm{~cm}$ & $10 \mathrm{~cm}$ & $20 \mathrm{~cm}$ & $1 \mathrm{~cm}$ & $10 \mathrm{~cm}$ & $20 \mathrm{~cm}$ & $1 \mathrm{~cm}$ & $10 \mathrm{~cm}$ & $20 \mathrm{~cm}$ \\
\hline Axial & 3.96 & 4.83 & NA & 8.37 & 9.45 & $N A$ & 4.14 & 4.72 & 5.81 & 8.51 & 9.30 & 11.76 \\
\hline Transverse (radial) & 4.11 & 4.47 & NA & 8.38 & 8.72 & $N A$ & 4.01 & 4.64 & 5.83 & 8.37 & 8.97 & 10.41 \\
\hline Transverse (tangential) & 4.11 & 4.38 & NA & 8.38 & 8.94 & $N A$ & 4.01 & 4.41 & 4.95 & 8.37 & 8.91 & 10.43 \\
\hline
\end{tabular}

Spatial resolution is measured in millimeters with radial offset at $1 \mathrm{~cm}, 10 \mathrm{~cm}$, and $20 \mathrm{~cm}$

${ }^{a}$ Measurement 01 contains all tests except for the radial offset at $20 \mathrm{~cm}$; we repeated all test including the $20 \mathrm{~cm}$ offset results as shown in measurements 02

Figure 5 summarizes count loss measurements of the dPET as a function of activity concentration. With the amount of $\sim 1100 \mathrm{MBq}(\sim 30 \mathrm{mCi}){ }^{18} \mathrm{~F}$ used for the count loss test, neither the peak true count rate nor the peak NECR could not be found as the system did not show a peak value beyond which the NECR began to decrease with increasing activity. Consequently, in this study, we use 'maximum' instead of 'peak' to describe these results. It revealed a maximum NECR of $171 \mathrm{kcps}$ at $50.5 \mathrm{kBq} / \mathrm{mL}$, with corresponding true count rate of $681 \mathrm{kcps}$ and scatter fraction of $30.8 \%$ at the maximum NECRs, respectively.
NEMA image quality of the IEC body phantom PET reconstructed in HD using 3D OSEM with point spread function (PSF) is shown in Fig. 6a. Image contrast recovery and background variability are given in Fig. $6 \mathrm{~b}$ and c. The average lung error was $4.18 \pm 0.05 \%$.

Results of timing and energy resolution of the dPET system are shown in Fig. 7. Figure 7a demonstrates the NEMA NU2-2018 coincidence timing resolution as a function of activity concentration which was measured as 332 ps in FWHM and 640 ps in FWTM, with robust distribution $(1 \pm 2 \%$ for FWHM and $2 \pm 1 \%$ for FWTM)

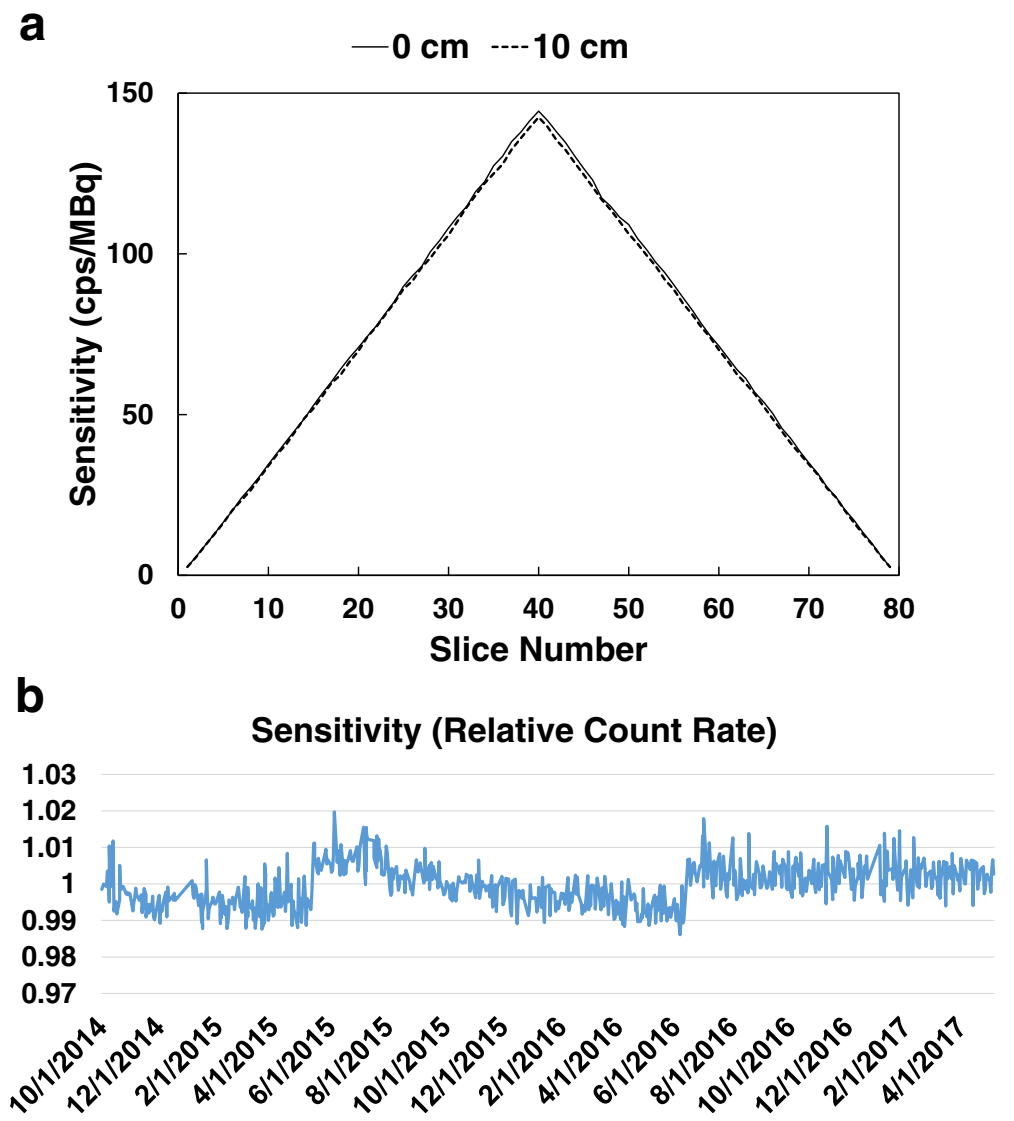

Fig. 4 NEMA sensitivity of the dPET system with (a) NEMA sensitivity profile as a function of axial slices and (b) sensitivity stability over 31 month (<1.5\% variability) with two jump points in June 2015 and July 2016 after system recalibration 

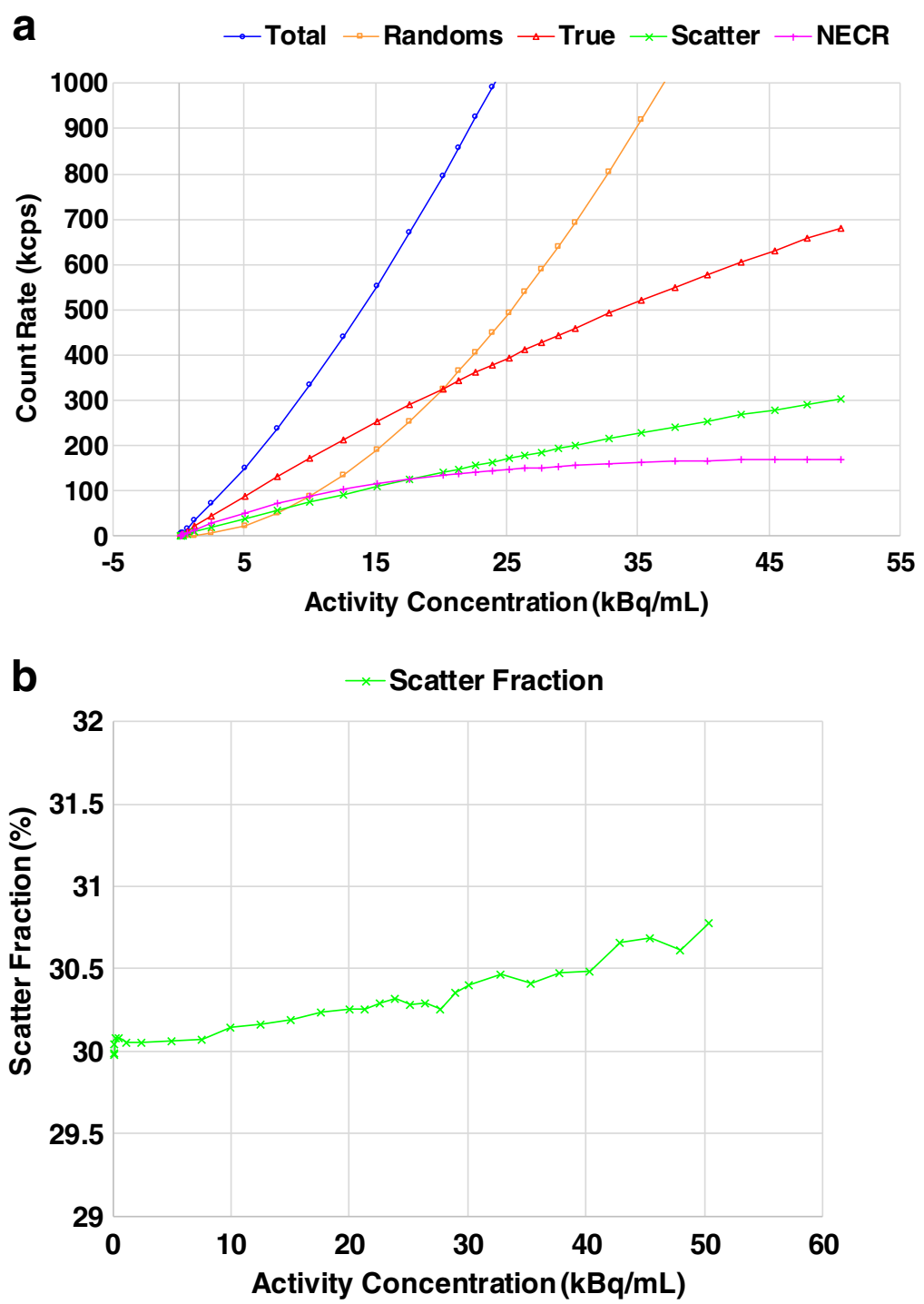

Fig. 5 NEMA count rate measurements as a function of activity concentration. a Count rate of total, randoms, true, and NECR. b Scatter fraction

obtained as increasing activity concentration from 0 to $50.5 \mathrm{kBq} / \mathrm{mL}$. Fig. $7 \mathrm{~b}$ and c shows the stability of timing and energy resolution $\left({ }^{22} \mathrm{Na}\right.$ point source based) within the monitoring time window. It revealed $<1 \%$ variability for timing and $<0.5 \%$ variance for energy. The average energy resolution was about $11.2 \%$ FWHM.

\section{Additional phantom measurements}

Figure 8 summarizes dPET image quality using different phantom measurements. dPET images of the ACR phantom is shown in Fig. 8a. With the reduced PVE using smaller voxels, the smallest $4.8 \mathrm{~mm}$ rods are detectable on both HD and UHD dPET. Fig. 8b shows the CQIE uniformity of dPET (256 mm FOV) giving $<3 \%$ axial variation in SUV which is well below the CQIE 10\% acceptable threshold. Images of the IEC body phantom varying with SBRs are compared between dPET and cPET shown in Fig. 8c. Increasing SBR to 16 and above, deformation of the spheres and artifacts for the lung insert appeared to be more and more visible on cPET but not dPET images, and better sphere delineation with enhanced contrast adapting to different SBRs was found on dPET (the potential reason is given in the "Discussion" section). Quantitatively, it indicated an average differences of $(-7 \pm 4 \%,-6 \pm 5 \%,-5 \pm 5 \%,-$ $6 \pm 5 \%,-22 \pm 8 \%$, and $-28 \pm 12 \%$ ) for spheres of (37, $28,22,17,13$, and $10 \mathrm{~mm})$ over all SBRs, and an average differences of $(-3 \pm 4 \%,-10 \pm 7 \%,-14 \pm 10 \%$, $-7 \pm 12 \%,-16 \pm 11 \%,-19 \pm 13 \%$, and $-18 \pm 15 \%)$ for 


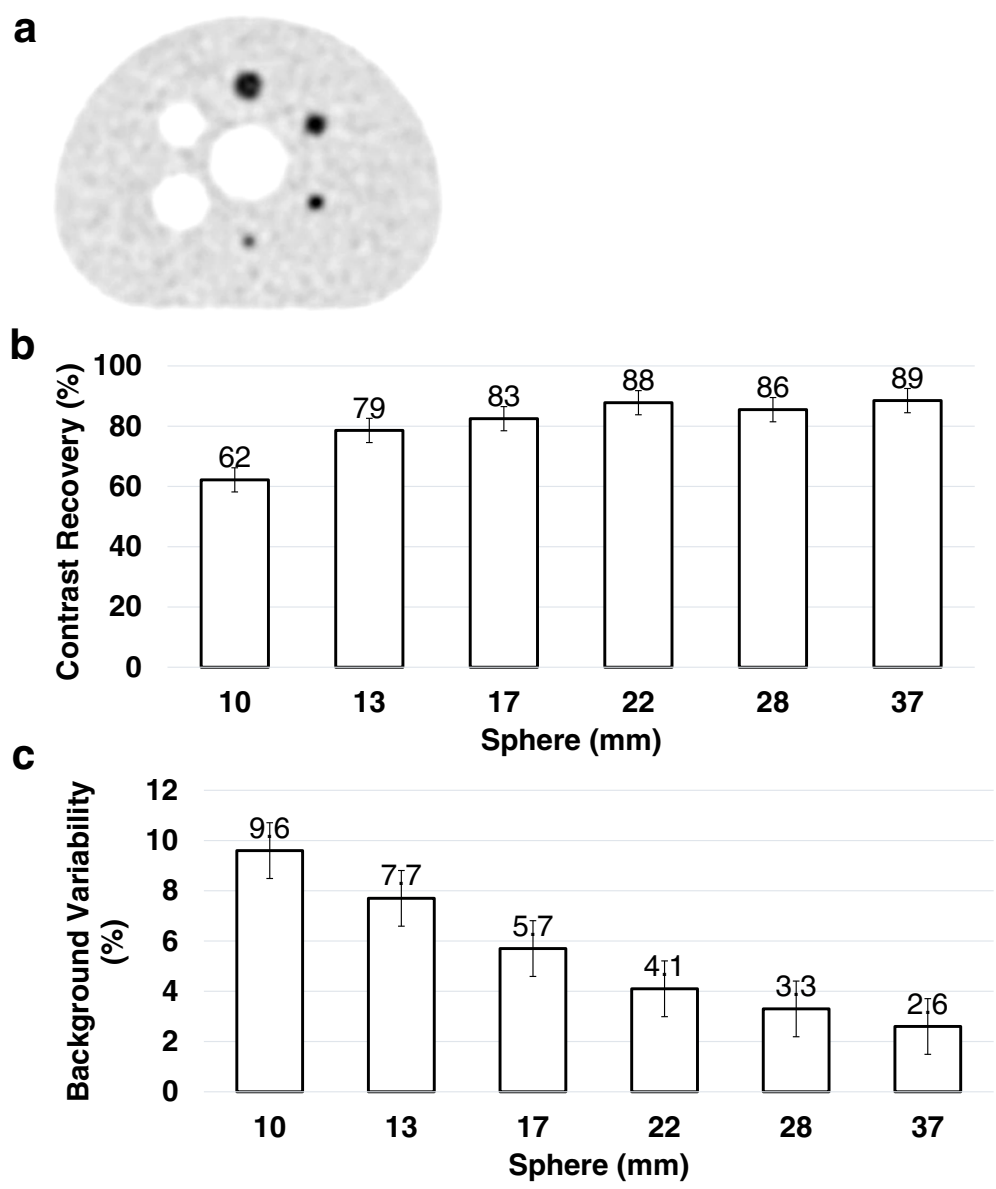

Fig. 6 NEMA NU2-2012 image quality of the IEC body phantom PET reconstructed using 3D OSEM TOF PSF. a PET image (b and $\mathbf{c}$ ), assessment of contrast recovery, and background variability, respectively. The average lung error was $4.18 \pm 0.05 \%$

SBRs of $(2,4,8,16,32,64$, and 100) over all spheres, for $\mathrm{RC}_{\max }$ of $\mathrm{CPET}$ compared to $\mathrm{dPET}$.

\section{Clinical patient studies}

While patient studies are not the focus of this paper, Fig. 9 presents an intra-individual comparison between cPET and dPET of the same clinical patient, which demonstrates improved lesion detectability with better contrast of lesions on the 322 ps dPET compared to the 550 ps cPET.

Figure 10 presents a clinical FDG case scanned on the dPET system and reconstructed using SD, HD, and UHD algorithms by isotropically reducing voxel sizes in consistent to the EARL effort using the DPC system [38]. Dramatically enhanced lesion visibility with improved diagnostic confidence using blinded reader studies [39] was found on HD (8 $\mathrm{mm}^{3}$ voxel) and particularly UHD $\left(1 \mathrm{~mm}^{3}\right.$ voxel $)$ dPET images.

\section{Discussion}

dPET enables every digital photon counter detector to be directly coupled to a single scintillator and leads to higher count rate detection efficiency across a more uniform scintillator distribution compared to CPET. The individual DPC detector controlling greatly reduces the impact of count rate dependent pile-up on energy and timing resolution, and provides highly stable performance across a large dynamic range of count rates.

The major improvement of the dPET system is its significantly improved TOF timing resolution. The benefit of TOF PET has been well-established and became a benchmark technology $[40,41]$. Current cPET has timing resolution of $400-600 \mathrm{ps}$ with a scintillator length range of $12-25 \mathrm{~mm}$ [42-50]. With the introduction of SiPM-based photo detection technologies, the available solid-state PET systems are achieving timing resolution in the range of $300-400$ ps $[21,51]$, with the investigated dPET demonstrating 322 ps (Table 2). The 322 ps TOF approximately halves the uncertainty from 600 ps TOF PET systems, limits the noise contribution from fewer voxels during PET reconstruction, improves image SNR, and converges faster in 3D-OSEM TOF reconstructions necessitating less 


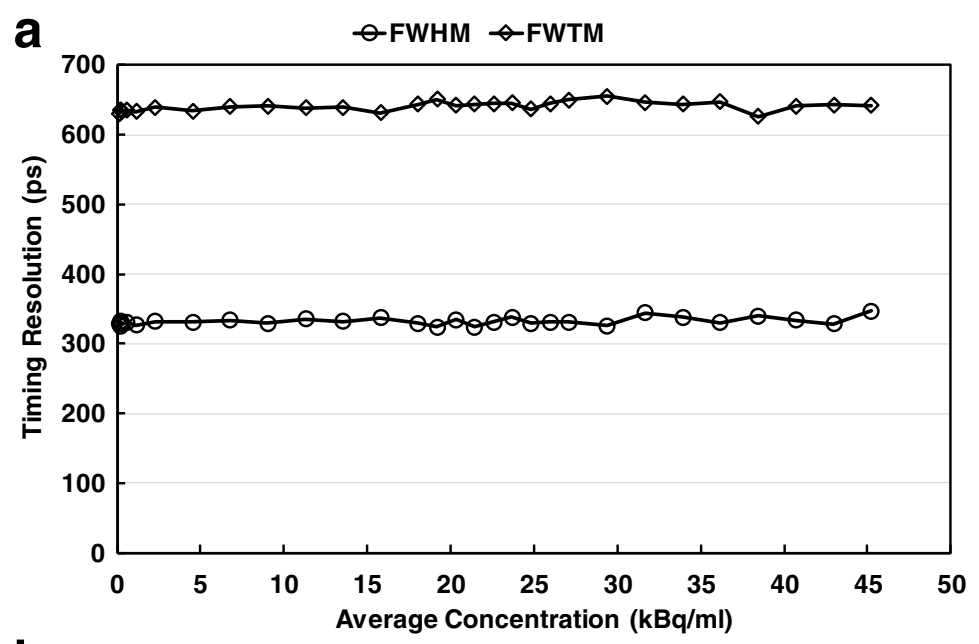

b

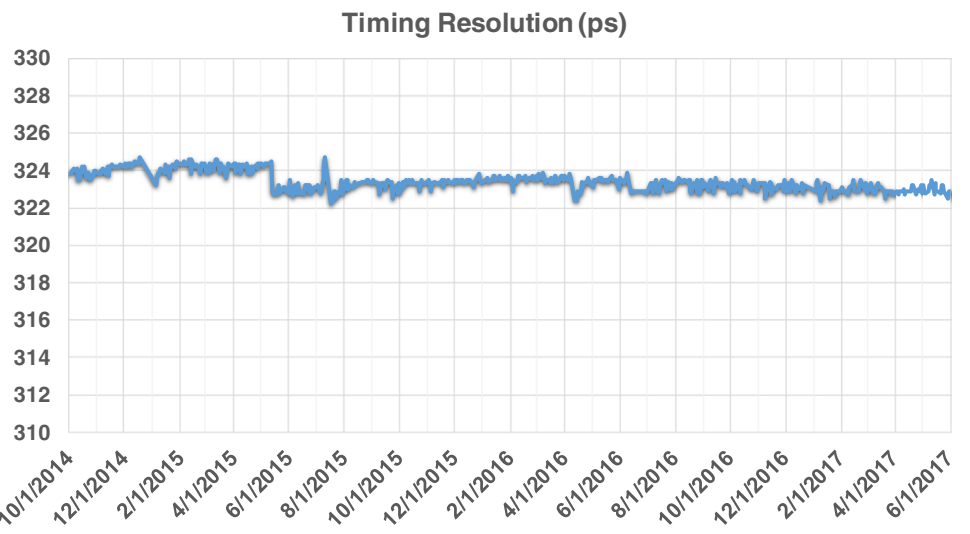

C

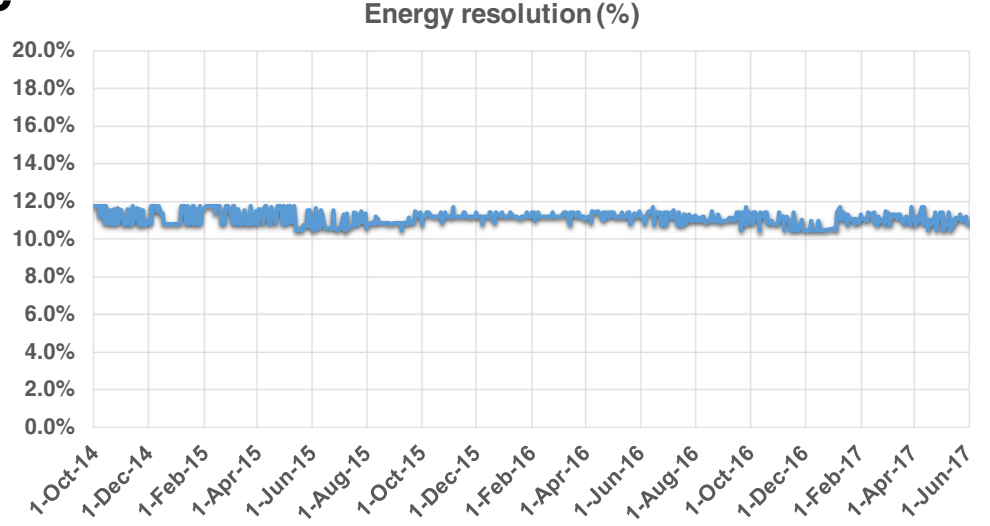

Fig. 7 Timing resolution and energy resolution of the dPET system. a NEMA NU2-2018 timing resolution in FWHM and FWTM as a function of activity concentration. b Stability of timing resolution ( ${ }^{22} \mathrm{Na}$ point source based) within the 31-month monitoring time window. It demonstrates a slight improvement from 325 ps to 322 ps when system calibration was performed 6 months after installation. c Stability of energy resolution during the monitoring time period

iterative steps to achieve optimal image quality. In addition, the study used both the ${ }^{22} \mathrm{Na}$ point source (daily PET QC) and the NEMA NEC phantom data (NEMA NU2-2018 new standard) to determine the coincidence timing resolution. We found that the timing resolution measured using the point source based method is $\sim 10$ ps better than using the NEMA NEC phantom based methodology. To the best of our knowledge, this is potentially due to the vendor-specific software improvement in daily $\mathrm{QC}$ for the timing resolution 
a

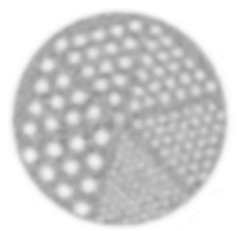

HD dPET

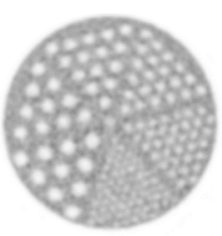

UHD dPET

b

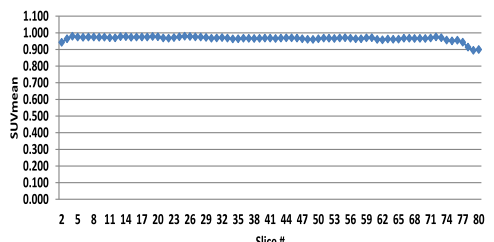

Slice \#

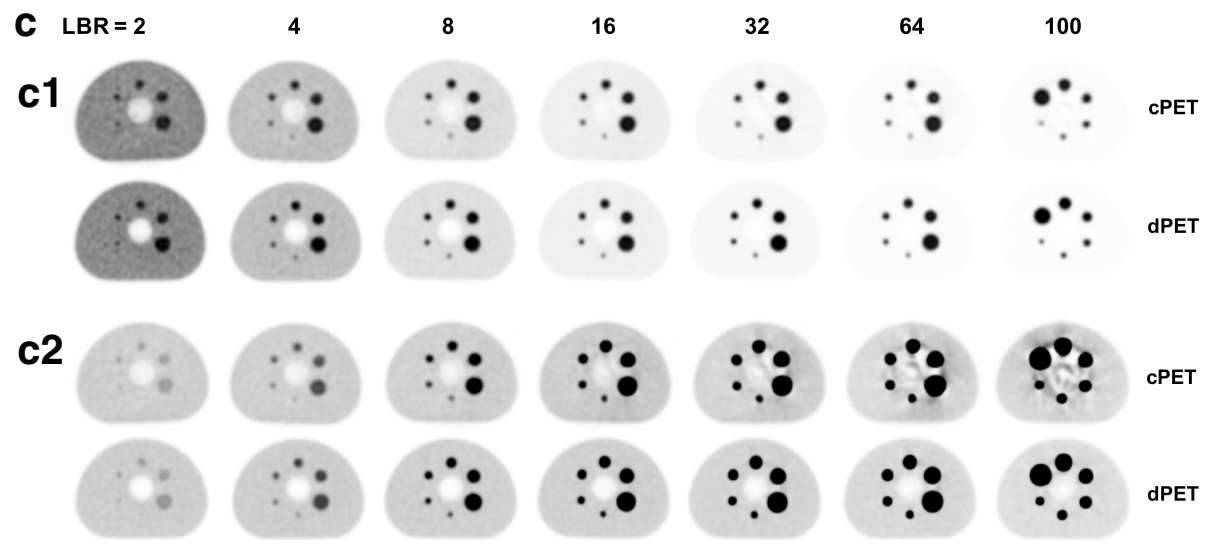

c3

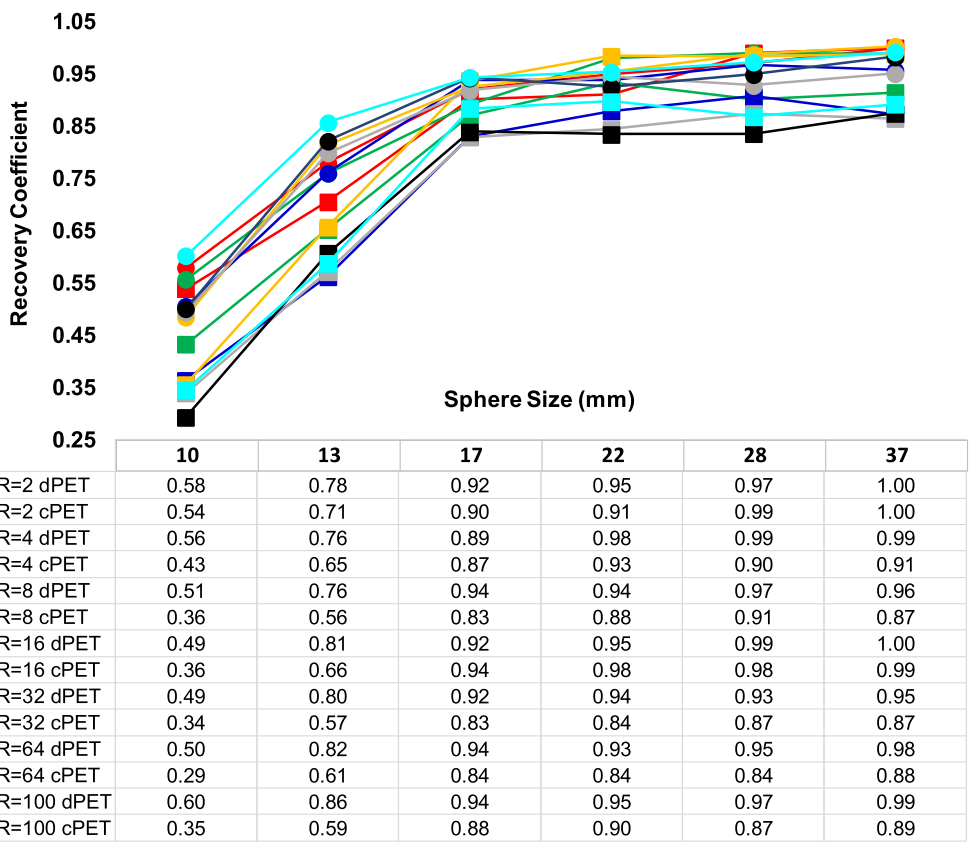

Fig. 8 Image quality of dPET via various phantom measurements. a HD and UHD dPET images of the ACR phantom cold rods. b CQIE dPET uniformity. c Comparison of dPET vs CPET of the NEMA IEC Body phantom images at different SBRs: (C1) window-level setting: SUV LL =0, $U L=S U V_{\text {max }}$ of CPET at each SBR; (c2) window-level setting: same background with SUV LL =0, UL = 5.0; (c3) RCS of dPET Vs CPET as a function of sphere sizes (10-37 mm) at different SBRs (2-100)

measurement, as well as the fact that the ${ }^{18} \mathrm{~F}$ line source in the NEMA NEC phantom is typically not perfectly straight.

Improvement of PET spatial resolution is another important feature of the dPET system. The fundamental limits of PET spatial resolution are impacted predominantly by crystal size in addition to positron range, non-collinearity, positioning decoding, and reconstruction methods. These factors limit the effective spatial resolution to different degrees. The spatial resolution can be parameterized as

$$
S R=K_{r} \times \sqrt{R_{i}^{2}+R_{p}^{2}+R_{a}^{2}+R_{l}^{2}}
$$

where $K_{r}$ is a factor of $1.2-1.5$ that resulted from 
a
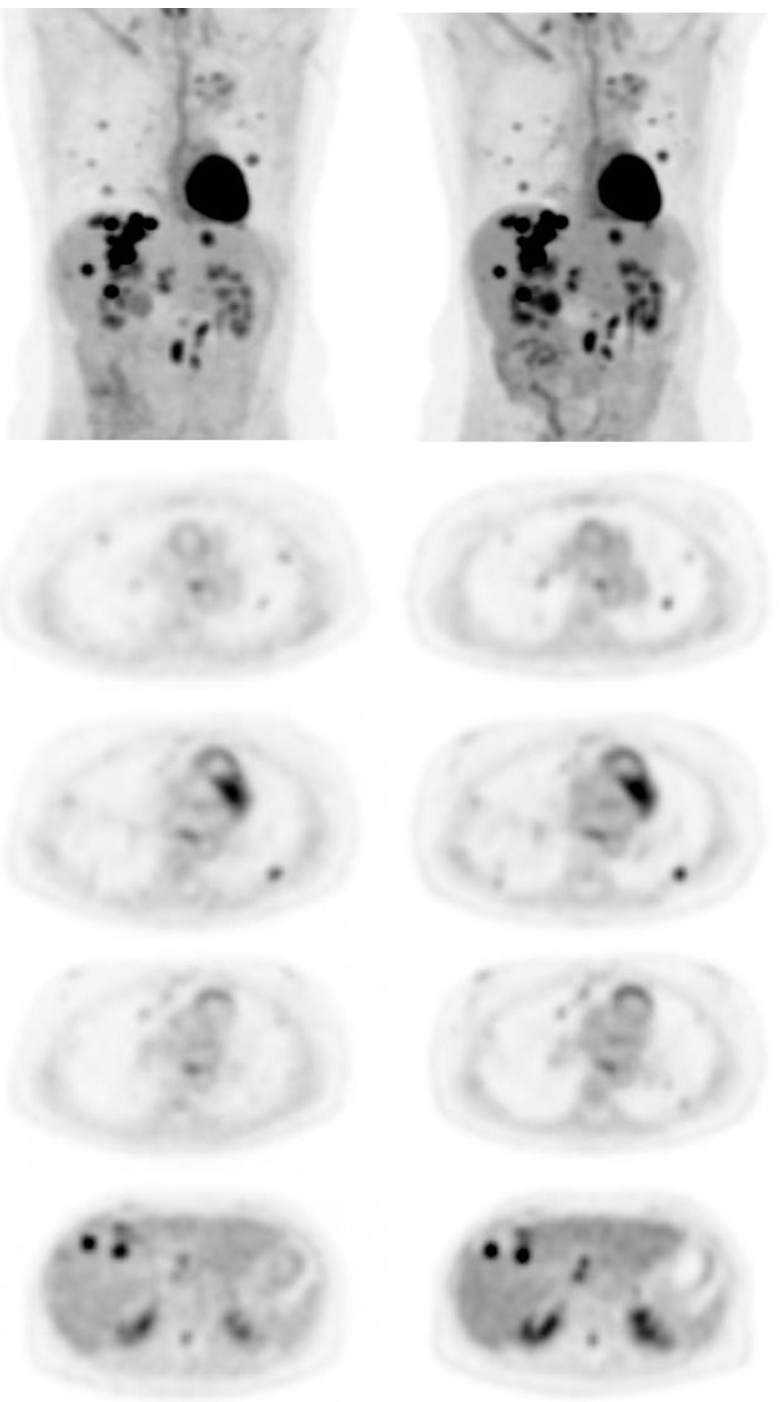

\section{L5}
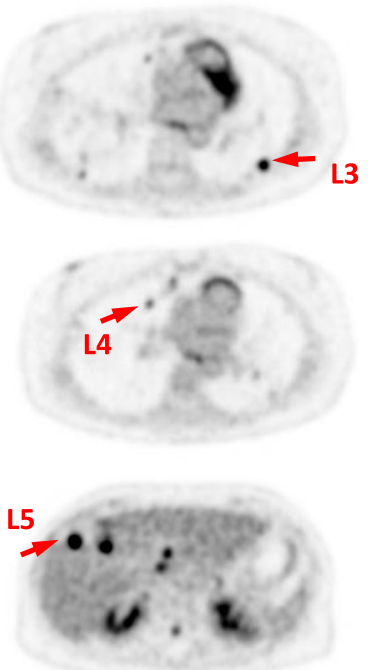

$\%$ increase
$49 \%$
$21 \%$
$8 \%$
$19 \%$
$17 \%$

dPET SD
1.03
1.21
2.59
1.05
5.33

$\%$ increase
$80 \%$
$50 \%$
$40 \%$
$65 \%$
$56 \%$

dPET UHD

1.24

1.50

3.35

1.45

7.12

Fig. 9 Intra-individual comparison of a SD CPET (75 min p.i., 3i33s) with b SD dPET (103 min p.i., 3i15s) and c UHD dPET (103 min p.i., $3 i 13 \mathrm{~s}$ ) for a clinical patient with extensive metastatic disease in the lung and the liver (BMI =18.5, $503 \mathrm{MBq} F D G$, $90 \mathrm{~s} / \mathrm{bed}$ ). Images were reconstructed using 3D OSEM TOF. The contrast of lesions is improved on SD dPET and prominently on UHD dPET compared to SD cPET. The lesion conspicuity and detectability as well as the diagnostic confidence for the pulmonary and hepatic lesions are classified by blinded reader analysis as substantially

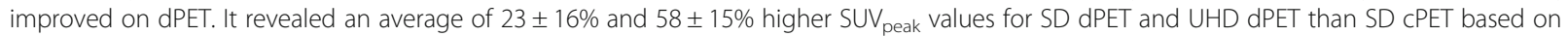
five representative lesions selected (pointed by red arrows), respectively

reconstruction methodologies [52], $R_{i}$ is the intrinsic resolution given by $d / 2$ with $d$ as the crystal width, $R_{p}$ is the error due to positron range $\left(\sim 0.2 \mathrm{~mm}\right.$ for $\left.{ }^{18} \mathrm{~F}\right), R_{a}$ is the error from PET non-collinearity given by $0.0022 D$ with $D$ as the ring diameter, and $R_{l}$ as the decoding error due to anger logic positioning localization [31, 53]. The dPET system with 1:1 coupling of scintillator and SiPM detector does not need anger logic position decoding 


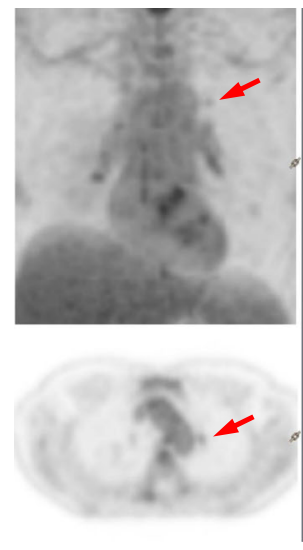

SD PET $\left(4 \times 4 \times 4 \mathrm{~mm}^{3}\right)$

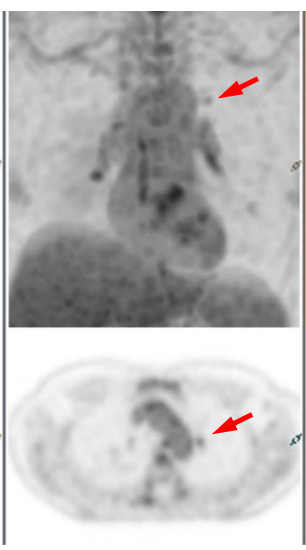

HD $\left(2 \times 2 \times 2 \mathrm{~mm}^{3}\right)$

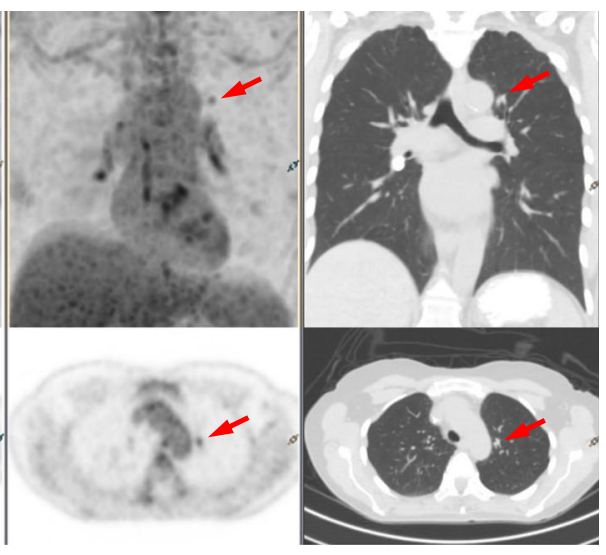

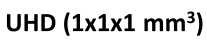

Fig. 10 Anal cancer patient scanned on the dPET system (BMI =24, 481 MBq FDG, 50 min uptake time) and reconstructed in SD, HD, and UHD using 3D OSEM TOF algorithm, in contrast with CT image on the right. The small lesion (red arrows) becomes more visible from SD PET to UHD PET with less PVE noted in the smaller isotropic voxel images

and therefore its degradation including distortions and edge effects is removed $\left(R_{l}=0\right) . K_{r}$ is adjusted to be 1.43-1.54 for the dPET system after applying our NEMA spatial resolution results onto the above formula. A 10$20 \%$ improvement of the NEMA spatial resolution compared to the cPET [42] was obtained. In addition, depth-of-interaction (DOI) corrections may further improve the precision of annihilation localization and spatial resolution; it is expected that DOI integration will be available in future dPET systems.

NEMA sensitivity of the dPET system was found to be less than its former PMT PET/CT systems (Ingenuity TF 64, Gemini TF 64). In 3D mode, PET sensitivity degrades from peak at center to both edges in axial direction and is related to axial FOV length, PET overlap, detector ring size, and scintillator length. Typically, smaller detector

Table 2 Comparison of system characteristics across manufacturer PET/CT systems

\begin{tabular}{|c|c|c|c|c|c|c|c|c|}
\hline Manufacture & GE & GE & Philips & Philips & Philips & Siemens & Siemens & Toshiba \\
\hline PET/CT model & $\begin{array}{l}\text { Discovery Ml } \\
\text { (4-ring) [51] }\end{array}$ & $\begin{array}{l}\text { Discovery } \\
690[43]\end{array}$ & $\begin{array}{l}\text { Vereos } \\
\text { (this work) }\end{array}$ & $\begin{array}{l}\text { Ingenuity } \\
\text { TF [44] }\end{array}$ & $\begin{array}{l}\text { Gemini } \\
\mathrm{T}[42]\end{array}$ & $\begin{array}{l}\text { Biograph } \\
\text { mCT flow [45] }\end{array}$ & $\begin{array}{l}\text { Biograph mCT } \\
{[46,47]}\end{array}$ & $\begin{array}{l}\text { Celesteion } \\
{[48-50]}\end{array}$ \\
\hline Photo detector & SiPM & PMT & SiPM & PMT & PMT & PMT & PMT & PMT \\
\hline Number of detectors & 9792 & 256 & 23,040 & 420 & 560 & 768 & 768 & 480 \\
\hline Scintillator & LYSO & LYSO & LYSO & LYSO & LYSO & LSO & LSO & LYSO \\
\hline Number of crystals & 19,584 & 13,824 & 23,040 & 28,336 & 28,336 & 32,448 & 32,448 & 30,720 \\
\hline Crystal size $\left(\mathrm{mm}^{3}\right)$ & $3.95 \times 5.3 \times 25$ & $4.2 \times 6.3 \times 25$ & $3.86 \times 3.86 \times 19$ & $4 \times 4 \times 22$ & $4 \times 4 \times 22$ & $4 \times 4 \times 20$ & $4 \times 4 \times 20$ & $4 \times 4 \times 12$ \\
\hline Ring diameter (cm) & 74.4 & 81.0 & 76.4 & 90.0 & 90.3 & 84.2 & 84.2 & 88.0 \\
\hline Axial FOV (cm) & 20.0 & 15.7 & 16.4 & 18.0 & 18.0 & 22.1 & 22.1 & 19.6 \\
\hline Plane spacing (mm) & $\mathrm{n} / \mathrm{a}$ & $\mathrm{n} / \mathrm{a}$ & 1,2 , or 4 & 2 or 4 & 2 or 4 & 2 & 2 & 2 \\
\hline TOF Timing resolution (ps) & 375 & 544 & 322 & 502 & 585 & 555 & 527 & 410 \\
\hline Sensitivity (cps/kBq) & 13.7 & 7.4 & 5.7 & 7.3 & 6.6 & 9.6 & 9.7 & 4.0 \\
\hline $\begin{array}{l}\text { Transverse resolution @ } \\
1 \mathrm{~cm}(\mathrm{~mm})\end{array}$ & 4.1 & 4.7 & 4.0 & 4.8 & 4.8 & 4.3 & 4.4 & 5.1 \\
\hline $\begin{array}{l}\text { Transverse resolution @ } \\
10 \mathrm{~cm}(\mathrm{~mm})\end{array}$ & 5.0 & 5.1 & 4.4 & 5.1 & 5.2 & 4.9 & 4.9 & 5.1 \\
\hline Axial resolution @1 cm (mm) & 4.5 & 4.7 & 4.0 & 4.7 & 4.8 & 4.3 & 4.4 & 5.0 \\
\hline Axial resolution @10cm (mm) & 6.0 & 5.6 & 4.8 & 5.2 & 4.8 & 5.9 & 5.7 & 5.4 \\
\hline Peak NECR (kcps @ kBq/mL) & $193.4 @ 21.9$ & $139.1 @ 29.0$ & $171 @ 50.5$ & $124.1 @ 20.3$ & $125 @ 17.4$ & $185 @ 29$ & $156 @ 31.1$ & $\geq 51 @ n / a$ \\
\hline Energy resolution (\%) & 9.4 & 12.4 & 11.2 & 11.1 & 11.5 & $\mathrm{n} / \mathrm{a}$ & 11.5 & 11.3 \\
\hline $\begin{array}{l}\text { Scatter fraction at peak NECR } \\
(\%)\end{array}$ & 40.6 & 37 & 30.8 & 36.7 & 27 & 33.4 & 32.7 & 42.7 \\
\hline
\end{tabular}


rings with larger axial FOV lead to higher sensitivity scanners, and a PET overlap of $50 \%$ maintains a near uniform sensitivity at peak level. Compared to the prior CPET, the dPET system reduced the axial FOV length from $180 \mathrm{~mm}$ to $164 \mathrm{~mm}$, the PET acquisition overlap from $53 \%$ to $39 \%$, and the number of crystals by about $20 \%$ which in overall led to a reduced NEMA sensitivity from 7.3 (cPET) to $5.7 \mathrm{cps} / \mathrm{kBq}$ (dPET). The question to be asked is why reducing the system sensitivity by $\sim 22 \%$ does not interfere with lesion detection but conversely has enabled improved image quality and lesion detectability of the dPET. This observation is consistent to the reduction of sensitivity resulting from a sparse-ring configuration [54]. From the sensitivity point of view, NEMA sensitivity measures only a system's ability to convert photons to raw counts and does not take into account the quality of counts, and its reduction leads to fewer counts in quantity instead of quality. In another words, obtaining high-quality counts is more important than obtaining more lower-quality counts with a higher uncertainty, such as that the PSF resolution recovery curve of PMT systems tend to show long tails caused by 'bad' counts. For the dPET system, the SNR gain [55] from improved TOF timing resolution more than compensates for the somewhat reduced system sensitivity, and compared to non-TOF it translates NEMA sensitivity into an "effective sensitivity" improvement which contributes to 'good' counts instead of 'more' counts, as follows

$$
\begin{aligned}
& S_{\mathrm{eff}}(A)=S_{\mathrm{NEMA}}(A) \times G_{\mathrm{TOF}} / f_{d} \\
& G_{\mathrm{TOF}}=\frac{D}{\Delta x}=\frac{2 D}{c \Delta t}
\end{aligned}
$$

where $S_{\text {eff }}$ is the activity-dependent effective sensitivity,
$S_{\text {NEMA }}$ is the NEMA sensitivity, and $G_{\text {TOF }}$ is the TOF gain defined as the object diameter $(D)$ divided by the TOF localization uncertainty with $c$ the speed of light and $\Delta t$ the timing resolution, and $f_{d}$ the dead time correction factor. The 322 ps dPET system presented an effective sensitivity with a gain of 4.1 for thin body size or brain PET (20 cm diameter), 6.2 for average body size $(30 \mathrm{~cm})$, and 8.3 for large body size $(40 \mathrm{~cm})$ acquisitions, which contribute to better images of high-quality counts, although the NEMA sensitivity is lower. An example of effective sensitivity comparing dPET with cPET is shown in Fig. 11. A potential advantage of the dPET system which may generate good image quality under low sensitivity

Image noise is usually characterized by NECR which is proportional to SNR and frequently used to compare the performance between different PET systems. In this study, even though it is not clear whether or not the measured maximum NECR of $171 \mathrm{kcps}$ at $50.5 \mathrm{kBq} / \mathrm{mL}$ is the peak NECR, it seems that the plateau appears around this level. In addition, the dPET system with the introduced 1:1 coupling and vastly increased number of signal processing units allows it to handle far more counts per unit time than traditional PMT systems, which technically translates to a much higher peak true count rate. Despite using higher than usual ${ }^{18} \mathrm{~F}$ activity of $\sim 1100 \mathrm{MBq}$ for the NEMA count loss test, the testing failed to disclose the peak true count rate and its peak activity concentration of the dPET system. In our recent NEMA test using $>3000 \mathrm{MBq}{ }^{18} \mathrm{~F}(>80 \mathrm{mCi}$, ongoing different project and not included in this work), the system peak true count rate appears to be close to $900 \mathrm{kcps}$

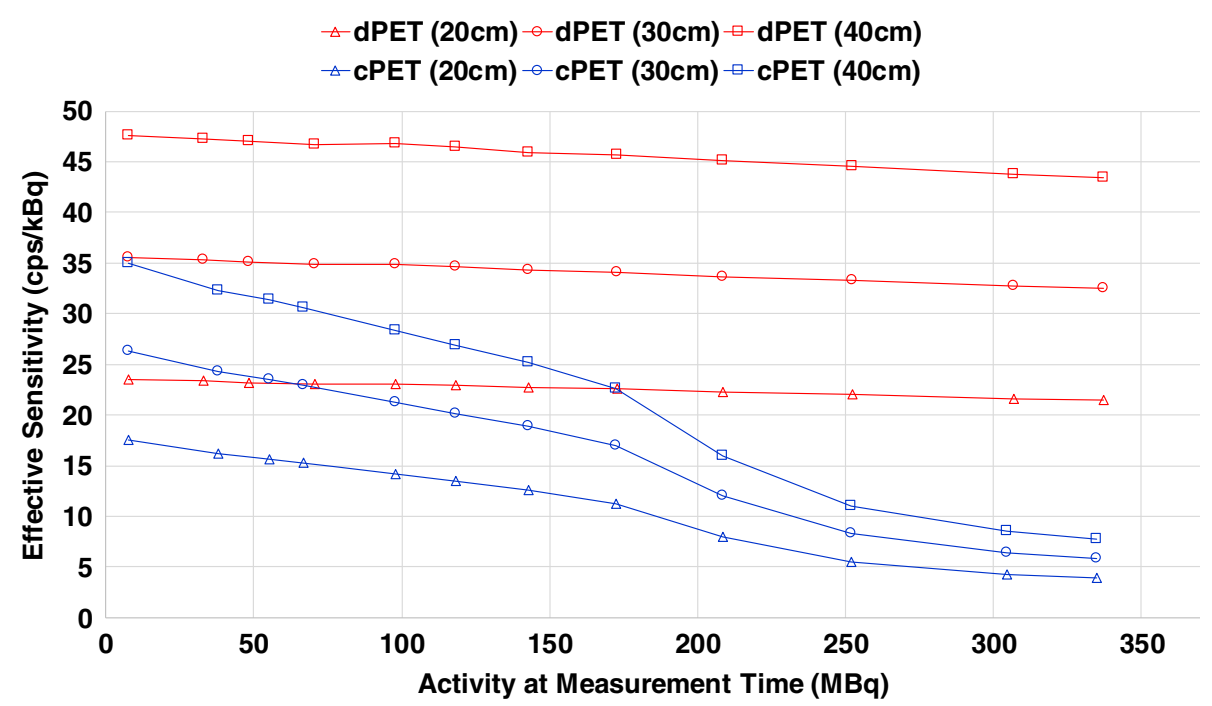

Fig. 11 Effective sensitivity of dPET (322 ps) versus CPET (550 ps) as a function of ${ }^{18}$ F-FDG activities for object diameters of 20, 30, and $40 \mathrm{~cm}$. It indicates a 1.3-5.5x effective sensitivity improvement of APET with improved quality of counts compared to CPET over the measured activity of 7.4-337 MBq ${ }^{18} \mathrm{~F}-\mathrm{FDG}$, although the NEMA sensitivity of dPET is lower than CPET's 
at $\sim 85 \mathrm{kBq} / \mathrm{mL}$. This ultra-wide dynamic range of count rates can hopefully provide significant benefits to high count rates acquisitions such as cardiac perfusion PET.

Some factors may contribute to the appearance of artifacts on the cPET compared to the dPET at high SBRs in Fig. 8c. First of all, it is difficult to visually identify the existing artifacts and deformation on the phantom CPET images if using a wider SUV window to display, while they become apparently visible when the window-level settings are calibrated on similar background levels (e.g., 0-5 SUV), as shown in Fig. 8c. Secondly, the NEMA NEC body phantom has a volume of $\sim 10 \mathrm{l}$ with a filled activity of $\sim 185 \mathrm{MBq}(\sim 5 \mathrm{mCi}){ }^{18} \mathrm{~F}$-FDG. This leads to an activity concentration of $\sim 18.5 \mathrm{kBq} / \mathrm{mL}$, which is high for the phantom particularly resulting in high count rate spheres when increasing SBRs. It asks for a system with larger dynamic range of count rate performance capability (such as NECR, the plateau @ $50.5 \mathrm{kBq} / \mathrm{mL}$ for the dPET compared to the peak @15.7 kBq/mL for the $\mathrm{CPET}$ ). Third, the timing resolution is very robust for the dPET (Fig. 7a) however has a big degradation for cPET system as increasing activity concentrations [42]. As a result, a substantial difference of effective NECR, which takes into consideration the TOF gain and the dead time correction factor, was found between both systems with a quick drop for the cPET ( 50\% drop of effective NECR from @15.7 kBq/mL to @ $25 \mathrm{kBq} / \mathrm{mL}$ ) compared to a continued rise for the dPET $(\sim 55 \%$ increase from @15.7 kBq/mL to @ $50.5 \mathrm{kBq} / \mathrm{mL}$ ). This may cause the changes of count rate behavior and count quality at the $\sim 18.5 \mathrm{kBq} / \mathrm{mL}$ activity concentration level.

While the broader clinical assessment is beyond the scope of this paper, we found in intra-individual comparison consistently improved image quality, detection, and classification of small lesions. It demonstrated that higher definition reconstruction appears to be of major clinical relevance for improved lesion detectability and characterization of lesion heterogeneity without increasing diagnostic ambiguity. It is expected that both the clinical sensitivity and specificity will be improved by dPET.

\section{Conclusions}

This study evaluated system performance of a new generation, solid-state digital photon counting PET/ CT representing the first such clinical system used for oncologic PET imaging. The system demonstrates excellent performance characteristics and stability over a 31-month monitoring period. Our findings reveal for the DPC PET clinical opportunities with improved image quality, lesion detectability and diagnostic confidence, and promising capabilities for performing faster and lower dose PET.

\section{Abbreviations}

APD: Avalanche photodiode; CPET: Conventional PMT-based PET/CT; CQIE: Center for Quantitative Imaging Excellence; DCR: Dark count rate; DOI: Depth-of-interaction; DPC: Digital photon counting; dPET: DPC PET/CT; FWHM: Full width at half-maximum; FWTM: Full width at tenth-maximum; HD: High definition; LYSO: Lutetiumyttrium oxyorthosilicate; MOS: Metal oxide semiconductor; NECR: Noise equivalent count rate; NEMA: National Electrical Manufacturers Association; OSU: The Ohio State University; PMT: Photomultiplier tubes; PVE: Partial volume effect; QC: Quality check; RC: Recovery coefficient; SBR: Sphere-to-background ratio; SD: Standard definition; SiPM: Silicon photomultiplier; SPAD: Single photon avalanche diode; SUV: Standardized uptake value; TOF: Time of flight; UHD: Ultra-high definition; WCIBMI: Wright Center of Innovation in Biomedical Imaging

\section{Acknowledgements}

Philips provided the clinical digital photon counting system for the investigational clinical trials prior to the commercial release of the system as part of a joint Ohio Third Frontier grant ODSA TECH 09-028. Technical support and feedback are greatly acknowledged from Dr. Michael Miller (Philips) and Dr. Yanfei Mao (Philips). We greatly appreciate all the help and support from Dr. Katherine Binzel (WCIBMI) in daily PET QC performance,

Dr. Michelle I. Knopp (WCIBMI) in the 10x dose reduction effort, Dr. Yu-Lung Hsieh (WCIBMI) in technical support, and Dr. Chadwick Wright (WCIBMI) for clinical case reviews.

\section{Funding}

This project was supported by the Ohio Third Frontier grants ODSA TECH 09-028 and ODSA TECH 13-060.

\section{Availability of data and materials}

All data generated or analyzed during this study are included in this published article.

\section{Authors' contributions}

JZ made substantial contributions to the conception and design of the work, experimental performance, data processing, analysis and interpretation, and manuscript write up. He provided final approval of the version submitted for publication and has agreed to be accountable for all aspects of the work in ensuring that questions related to the accuracy or integrity of any part of the work are appropriately investigated and resolved. PM made substantial contribution to the conception and design of the clinical trial and the lead on the investigational study from the side of the manufacturer. He contributed to the review and editing of the manuscript. MVK made substantial contributions to the conception and design of the work, clinical image reviews, guidelines, and manuscript write up. He provided final approval of the version submitted for publication and has agreed to be accountable for all aspects of the work in ensuring that questions related to the accuracy or integrity of any part of the work are appropriately investigated and resolved.

\section{Ethics approval and consent to participate}

The study (NCT02283125) was approved by the institutional review board, and all subjects signed an informed consent.

\section{Consent for publication}

The study (NCT02283125) was approved by the institutional review board, and all subjects signed an informed consent.

\section{Competing interests}

The first and senior authors declare that they have no competing interests. PM is an employee of Philips and Director of Clinical Science. He was the Philips co-PI of the clinical trials performed.

\section{Publisher's Note}

Springer Nature remains neutral with regard to jurisdictional claims in published maps and institutional affiliations.

\section{Author details}

${ }^{1}$ Wright Center of Innovation in Biomedical Imaging, Department of Radiology, The Ohio State University Wexner Medical Center, 395 W. 12th 
Avenue, Room 430, Columbus, OH 43210, USA. ${ }^{2}$ Philips Healthcare, Cleveland, OH, USA.

\section{Received: 20 July 2018 Accepted: 10 October 2018 Published online: 06 November 2018}

\section{References}

1. Mirzoyan R, Laatiaoui M, Teshima M. Very high quantum efficiency PMTs with bialkali photo-cathode. Nucl Instrum Methods Phys Res. 2006;567:230-2

2. Roncali E, Cherry SR. Application of silicon photomultipliers to positron emission tomography. Ann Biomed Eng. 2011:39(4):1358-77.

3. Daube-Witherspoon ME, Surti S, Perkins A, Kyba CCM, Wiener R, Werner ME, et al. The imaging performance of a LaBr3-based PET scanner. Phys Med Biol. 2010;55:45-64.

4. Peng Q, Choong WS, Vu C, Huber JS, Janecek M, Wilson D, et al. Performance of the tachyon time-of-flight PET camera. IEEE Trans Nucl Sci. 2015;62(1):111-9.

5. Son JW, Ko GB, Won JY, Yoon HS, Lee JS. Development and performance evaluation of a time-of-flight positron emission tomography detector based on a high-quantum-efficiency multi-anode photomultiplier tube. IEEE Trans Nucl Sci. 2016;63(1):44-51.

6. Gasanov AG. Soviet technical. Physics Letters Sov. 1988;14:313.

7. Bondarenko G, Buzhan P, Dolgoshein B, Smirnov K. Limited Geiger mode microcell photodiode: new results. Nucl Inst Methods. 2000;A442:187-92.

8. Golovin V, Saveliev V. Novel type of avalanche photodetector with Geiger mode operation. Nucl Inst Methods. 2004;A518:560-4.

9. Catana C, Wu Y, Judenhofer MS, Qi J, Pichler BJ, Cherry SR. Simultaneous acquisition of multislice PET and MR images: initial results with a MRcompatible PET scanner. J Nucl Med. 2006;47:1968-76.

10. Herbert DJ, Moehrs S, D'Ascenzo N, Belcari N, Del Guerra A, Morsani F. The silicon photomultiplier for application to high-resolution positron emission tomography. Nucl Instrum Methods Phys Res. 2007;A573:84-7.

11. Britvitch I, Johnson I, Renker D, Stoykov A, Lorenz E. Characterization of Geiger-mode avalanche photodiodes for medical imaging applications. Nucl Instrum Methods Phys. Res. 2007:A571:308-11.

12. McElroy DP, Saveliev V, Reznik A, Rowlands LA. Evaluation of silicon photomultipliers: a promising new detector for MR compatible PET. Nucl Instrum Methods Phys Res. 2007;A571:106-9.

13. Musienko Y, Auffray E, Lecoq P, Reucroft S, Swain J, Trummer J. Study of multi-pixelbecame avalanche photodiodes as a read-out for PET. Nucl Instrum Methods Phys Res. 2007;A571:362-5.

14. Renker D. New trends in photodetectors. Nucl Instrum Methods Phys Res. 2007;A71:1-6.

15. Lewellen TK. Recent developments in PET detector technology. Phys Med Biol. 2008;53(17):287-17.

16. Delso G, Furst S, Jakoby B, Ladebeck R, Ganter C, Nekolla SG, et al. Performance measurements of the Siemens mMR integrated whole-body PET/MR scanner. J Nucl Med. 2011:52(12):1914-22.

17. Th F, Prescher G, Degenhardt G, Gruyter R, Schmitz A, Ballizany R. The digital silicon photomultiplier - principle of operation and intrinsic detector performance. Nuclear Science Symposium Conference Record; 2009. p. N28-5.

18. Nguyen NC, Vercher-Conejero JL, Sattar A, Miller MA, Maniawski PJ, Jordan DW, et al. Image quality and diagnostic performance of a digital PET prototype in patients with oncologic diseases: initial experience and comparison with analog PET. J Nucl Med. 2015;56(9):1378-85.

19. Degenhardt C, Zwaans B, Frach T, Gruyter R. Arrays of digital silicon photomultipliers - intrinsic performance and application to scintillator readout. Nuclear science symposium conference record (NSS/MIC). 2010. IEEE. https://doi.org/10.1109/NSSMIC.2010.5874115.

20. Schaart DR, Charbon E, Frach T, Schulz V. Advances in digital SiPMs and their application in biomedical imaging. Nucl Inst Methods Phys Res A 2016;809:31-52.

21. Zhang J, Miller M, Knopp MV. Performance evaluation of digital PET/CT: medical physics basis for the clinical applications. Med Phys. 2016:43(6):3399

22. Wang W, Hu Z, Gualtieri EE, Parma MJ, Walsh ES, Sebok D, et al. Systematic and distributed time-of-flight list-mode PET reconstruction. Proc Nuclear Science Symp Conf Rec. 2006;3:1715-7.

23. Popescu LM, Lewitt RM. Ray tracing through a grid of blobs. 2004. IEEE. https://doi.org/10.1109/NSSMIC.2004.1466750.
24. Ye J, Song X, Hu Z. Scatter correction with combined single-scatter simulation and Monte Carlo simulation for 3D PET. 2014. IEEE. https://doi. org/10.1109/NSSMIC.2014.7431033.

25. Casey ME, Hoffman EJ. Quantitation in positron emission computed tomography. A technique to reduce noise in accidental coincidence measurements and coincidence efficiency calibration. J Comput Assist Tomogr. 1986;10:845-50

26. Richardson WH. Bayesian-based iterative method of image restoration. J Opt Soc Am. 1972:62:55-9.

27. Lucy LB. An iterative technique for the rectification of observed distributions. Astron J. 1974;79:745-65.

28. Zhang B, Olivier P, Lorman B, Tung C. PET image resolution recovery using PSF-based ML-EM deconvolution with blob-based list-mode TOF reconstruction. J Nucl Med. 2011;52(Supplement 1):266.

29. National Electrical Manufacturers Association. NEMA NU 2-2012 Performance Measurements of Positron Emission Tomographs (PETs). Rosslyn: National Electrical Manufacturers Association; 2013.

30. Matej S, Lewitt RM. 3D-FRP: direct Fourier reconstruction with Fourier reprojection for fully 3-D PET. IEEE Trans Nucl Sci. 2001;48(4):1378-85.

31. Saha GB. Performance Characteristics of PET Scanners. In: Basics of PET Imaging. New York: Springer; 2010.

32. Bailey DL, Jones T, Spinks TS. A method for measuring the absolute sensitivity of positron emission tomographic scanners. Eur J Nucl Med. 1991;18:374-9.

33. National Electrical Manufacturers Association. NEMA NU 2-2018: Performance Measurements of Positron Emission Tomographs (PET). Rosslyn: National Electrical Manufacturers Association; 2018.

34. Mao Y, Miller M, Bai C, et al. Evaluation of a TOF resolution measurement method using standard NEMA NEC phantom. J Nucl Med. 2017:58(supplement 1):436.

35. Wang GC, Li X, Niu X, Du H, Balakrishnan K, Ye H, et al. PET timing performance measurement method using NEMA NEC phantom. IEEE Trans Nucl Sci. 2016;63(3):1335-42.

36. Rosen M, Kinahan PE, Gimpel JF, Opanowski A, Siegel BA, Hill GC, et al. Performance observations of scanner qualification of $\mathrm{NCl}$-designated cancer centers: results from the centers of quantitative imaging excellence (CQIE) program. Acad Radiol. 2017;24(2):232-45.

37. MacFarlane CR. American College of Radiologists. ACR accreditation of nuclear medicine and PET imaging departments. J Nucl Med Technol. 2006:34(1):18-24.

38. Koopman D, Koerkamp MG, Jager PL, Arkies H, Knollema S, Slump CH, et al. Digital PET compliance to EARL accreditation specifications. EJNMMI Phys. 2017;4:9. https://doi.org/10.1186/s40658-017-0176-5.

39. Wright $\mathrm{CL}$, Binzel $\mathrm{K}$, Zhang J, Knopp MV. Advanced functional tumor imaging and precision nuclear medicine enabled by digital PET technologies. Contrast Media Mol Imaging. 2017;5260305. https://doi.org/10 1155/2017/5260305

40. Karp JS, Surti S, Daube-Witherspoon ME, Muehllehner G. The benefit of time-of-flight in PET imaging: experimental and clinical results. J Nucl Med. 2008;49(3):462-70

41. Conti M. Focus on time-of-flight PET: the benefits of improved time resolution. Eur J Nucl Med Mol Imaging. 2011;38(6):1147-57.

42. Surti S, Kuhn A, Werner ME, Perkins AE, Kolthammer J, Karp JS. Performance of Philips Gemini TF PET/CT scanner with special consideration for its timeof-flight imaging capabilities. J Nucl Med. 2007:48(3):471-80.

43. Bettinardi V, Presotto L, Rapisarda E, Picchio M, Gianolli L, Gilardi MC. Physical performance of the new hybrid PET/CT Discovery-690. Med Phys. 2011;38:5394-11.

44. Kolthammer JA, Su KH, Grover A, Narayanan M, Jordan DW, Muzic RF. Performance evaluation of the ingenuity TF PET/CT scanner with a focus on high count-rate conditions. Phys Med Biol. 2014:59(14):3843-59.

45. Rausch I, Cal-González J, Dapra D, Gallowitsch HJ, Lind P, Beyer T, et al. Performance evaluation of the biograph $\mathrm{mCT}$ flow PET/CT system according to the NEMA NU2-2012 standard. EJNMMI Phys. 2015;2:26.

46. Jakoby BW, Bercier Y, Conti M, Casey ME, Bendriem B, Townsend DW. Physical and clinical performance of the $\mathrm{mCT}$ time-of-flight PET/CT scanner. Phys Med Biol. 2011;56(8):2375.

47. Karlberg AM, Sæther O, Eikenes L, Goa PE. Quantitative comparison of PET performance-Siemens biograph mCT and mMR. Eur J Nucl Med Mol Imaging Phys. 2016;3:5. 
48. Burr KC, Wang G-CJ, Du H, Mann G, Balakrishnan K, Wang J, et al. A new modular and scalable detector for a time-of-flight PET scanner. IEEE Nuclear Science Symposium and Medical Imaging Conference (NSS/MIC); 2012. p. 2830-4.

49. Vandenberghe S, Mikhaylova E, D'Hoe E, Mollet P, Karp JS. Recent developments in time-of-flight PET. EJNMMI Physics. 2016;3:3.

50. Canon medical systems USA. https://us.medical.canon/products/computedtomography/celesteion/. Accessed 19 Oct 2018.

51. Hsu DFC, llan E, Peterson WT, Uribe J, Lubberink M, Levin CS. Studies of a next generation silicon-photomultiplier-based time-of-flight PET/CT system. J Nucl Med. 2017:58(9):1511-8.

52. Huesman RH. The effects of a finite number of projection angles and finite lateral sampling of projections on the propagation of statistical errors in transverse section reconstruction. Phys Med Biol. 1977;22:511.

53. Moses WW. Fundamental limits of spatial resolution in PET. Nucl Inst Methods Phys Res A. 2011;648:5236-40.

54. Zhang J, Knopp MI, Knopp MV. Sparse detector configuration in SiPM digital photon counting PET: a feasibility study. Mol Imaging Biol. 2018. https://doi. org/10.1007/s11307-018-1250-7.

55. Budinger TF. Time-of-flight positron emission tomography: status relative to conventional PET. J Nucl Med. 1983;24(1):73-8.

\section{Submit your manuscript to a SpringerOpen ${ }^{\circ}$ journal and benefit from:}

- Convenient online submission

- Rigorous peer review

- Open access: articles freely available online

High visibility within the field

- Retaining the copyright to your article

Submit your next manuscript at $\boldsymbol{\nabla}$ springeropen.com 